\title{
Nematicidal, Larvicidal and Antimicrobial Activities of Some New Mannich Base Imidazole Derivatives
}

\author{
Xiangxiong Chen ${ }^{1 *}$, Seung Woo Lee ${ }^{1}$, Akbar Idhayadhulla ${ }^{2}$, Radhakrishnan \\ Surendra Kumar ${ }^{3}$ and Aseer Manilal ${ }^{4}$ \\ ${ }^{1}$ School of Chemical Engineering, Yeungnam University, Gyeongsan, South Korea, ${ }^{2}$ Department of Chemistry, School of Basic \\ Sciences, Vels Institute of Science, Technology \& Advanced Studies - VELS University, Chennai-600117, Tamil Nadu, India; \\ ${ }^{3}$ Department of Chemistry, Shivani Engineering College, (Affiliated to Anna University), Tiruchirappalli, Tamil Nadu, India; \\ ${ }^{4}$ Department of Medical Laboratory Sciences, College of Medicine and Health sciences, Arba Minch University, Arba Minch, \\ Ethiopia
}

*For correspondence: Email: c.xiangxiong@yahoo.com

\begin{abstract}
Purpose: To synthesize Mannich base imidazole derivatives, and evaluate their antimicrobial, nematicidal and larvicidal properties .

Methods: Compounds 1a-g and 2a-g were prepared using a Mannich condensation method. The chemical structures of compounds 2a-g were confirmed by Fourier transform infrared spectroscopy (IR), proton nuclear magnetic resonance ( $\left.{ }^{1} \mathrm{H}-\mathrm{NMR}\right)$, carbon nuclear magnetic resonance $\left({ }^{13} \mathrm{C}-\mathrm{NMR}\right)$, and mass spectrometry (MS) and elemental analyses. Compounds 1a-f and 2a-f were screened for antimicrobial properties using an agar diffusion method. The nematicidal activity of the compounds was evaluated against juvenile Meloidogyne javanica as test organism while larvicidal activity was assessed against the urban mosquito, Culex. Quinquefasciatus, using a standard bioassay protocol.

Results: Compounds $1 \mathrm{~b}, 1 \mathrm{~g}, 2 \mathrm{e}$ and $2 \mathrm{~g}$ were highly active against a few bacterial organisms compared with the reference antibacterial, ciprofloxacin while the antifungal activity of compound $2 d$ was high compared with the reference, clotrimazole. Compounds $1 \mathrm{c}, 1 \mathrm{e}, 1 \mathrm{~g}$, and $2 \mathrm{e}$ showed high toxicity levels of larvicidal activity based their half maximal lethal dose $\left(L D_{50}\right)$ values. Compounds $1 d, 1 e, 1 f, 1 g, 2 d$ and 2e were highly toxic to nematodes.

Conclusion: Compounds $1 \mathrm{~b}, 1 \mathrm{~g}, 2 \mathrm{e}$ and $2 \mathrm{~g}$ may be useful as lead molecules for the development of new classes of larvicidal, nematicidal and antimicrobial agents.
\end{abstract}

Keywords: Imidazole, Thiosemicarbazide, Semicarbazide, Condensation, Antimicrobial, Nematicidal, Larvicidal, Structure-activity relationship.

Tropical Journal of Pharmaceutical Research is indexed by Science Citation Index (SciSearch), Scopus, International Pharmaceutical Abstract, Chemical Abstracts, Embase, Index Copernicus, EBSCO, African Index Medicus, JournalSeek, Journal Citation Reports/Science Edition, Directory of Open Access Journals (DOAJ), African Journal Online, Bioline International, Open-J-Gate and Pharmacy Abstracts

\section{INTRODUCTION}

Culex is an important genus of mosquito that acts as a vector for several serious diseases, including filariasis, west Nile virus, dangue fever, yellow fever, chikungunya and other diseases caused by encephalitides. Nematodes are tiny worms, some of which are plant parasites. It is thought that nematode infections play an important role in the predisposition of a host plant to invasion by secondary pathogens. Edible plants attacked by nematodes show retarded growth and development, resulting in loss of quality and quantity of the harvest.

Currently employed nematicides are slated for tighter regulations and less use due to environmental problems and human and animal health concerns. The optimum methods of 
controlling mosquito larvae and nematodes involve the use of insecticides such as various organophosphates, and natural and synthetic heterocyclic products. New environmentally safe and biodegradable insecticides that specifically target mosquitoes and nematodes are urgently needed.

Naturally occurring and synthetic imidazole derivatives are an important class of heterocycles that are known to exhibit various biological activities [1]. The imidazole nucleus is a major component in a variety of drugs, including angiotensin II receptor antagonists, anti-inflammatory agents, protein kinase inhibitors, and fungicides [2]. Imidazole also plays important roles in biochemical processes [3]. Many substituted imidazoles are used as fungicides and herbicides, plant growth regulators and therapeutic agents [4]. Imidazole is common compounds of a large number of biologically and medicinally significant substances [5,6], including anticonvulsant [7] and monoamine oxidase (MAO) inhibitors [8].

The Mannich reaction is commonly employed to develop agricultural chemicals such as plant growth regulators [9] and is an important tool in the synthesis and modification of biologically active compounds [10]. It provides a convenient access to many useful synthetic building blocks because amino groups can be easily converted into a variety of other functionalities [11]. Mannich bases often exhibit significant biological properties including antimicrobial [12], cytotoxic [13] and anticancer [14].

The present investigation focuses on a series of imidazole compound in a single molecular framework and examines their larvicidal, nematicidal, antibacterial and antifungal

\section{EXPERIMENTAL}

\section{Materials}

All the melting points were recorded in open capillary tubes and are uncorrected. IR spectra were recorded in $\mathrm{KBr}$ on a $\mathrm{FT}-\mathrm{IR}$ Shimadzu $8201 \mathrm{pc}$ and ${ }^{1} \mathrm{H}$ NMR spectra were recorded on a Brucker DRX-300 MHZ. Elemental analysis (C, H and $\mathrm{N}$ ) were undertaken using an Elementer analyzer model vario EL III. The purity of the compounds was checked by thin layer chromatography (TLC) with silica gel plates.

General procedure for the synthesis of 2-[1Himidazol-1-yl (phenyl) methyl] hydrazinecarbo thioamide (1a)

Imidazole $(0.1 \mathrm{~mol}), \quad$ thiosemicarbazide hydrochloride $(0.1 \mathrm{~mol})$, and benzaldehyde $(0.1$ $\mathrm{mol})$ were added in ethanol solvent $(20 \mathrm{~mL})$. The reaction mixture was refluxed $5 \mathrm{~h}$ with temperature $60{ }^{\circ} \mathrm{C}$. Then the mixture was poured over crushed ice. The precipitate was obtained in few min. then the precipitate was collected by filtration. The precipitate was dried and recrystallized from suitable alcohols. The above procedure was followed by all the remaining compounds 1a-1g.

\section{2-[1H-imidazol-1-yl (phenyl) methyl] hydrazinecarboxamide (2a)}

Imidazole, $(0.1 \mathrm{~mol})$, semicarbazide hydrochloride $(0.1 \mathrm{~mol})$ and benzaldehyde $(0.1 \mathrm{~mol})$ were added in ethanol solvent $(20 \mathrm{~mL})$. The reaction mixture was refluxed $5 \mathrm{~h}$ with $60{ }^{\circ} \mathrm{C}$. Then the mixture was poured over crushed ice. The precipitate was obtained in few min. then the precipitate was collected by filtration. activities.

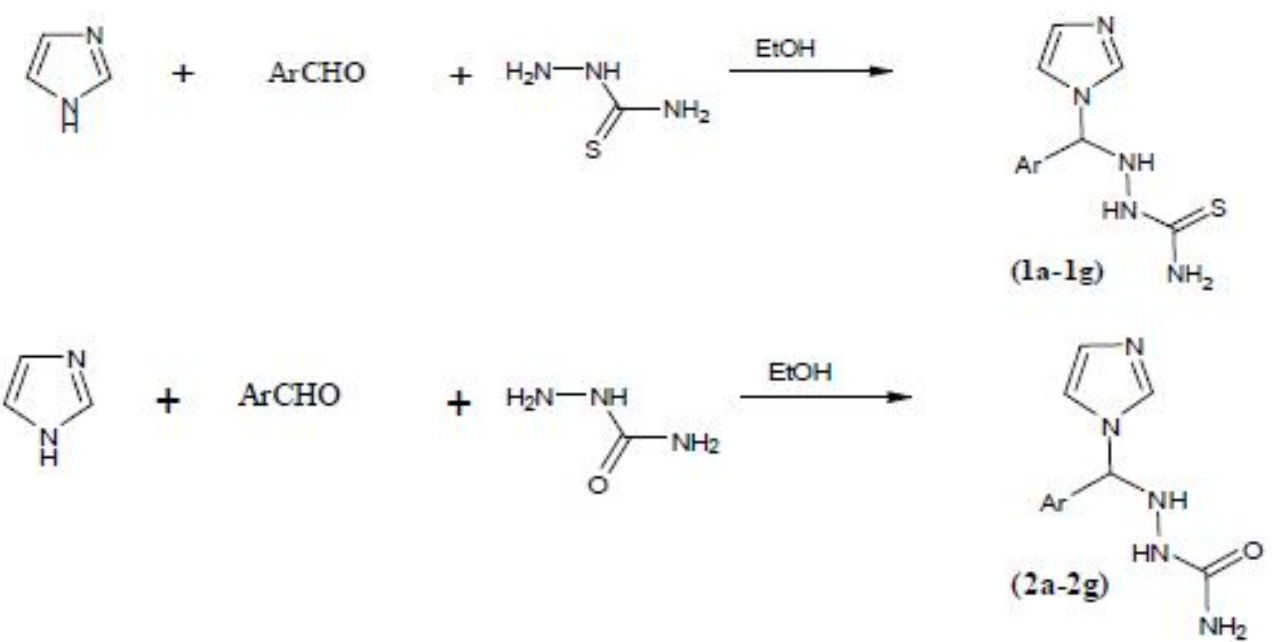

Scheme 1: Synthetic route of compounds $1 \mathrm{a}-1 \mathrm{~g}$ and $\mathbf{2 a - 2 g}$ 
The precipitate was dried and recrystallized from suitable alcohols. The above procedure was followed by all the remaining compounds $\mathbf{2 a - 2} \mathbf{g}$.

\section{In vitro antibacterial screening}

Compounds $\mathbf{1 a - 1 g}$ and $\mathbf{2 a}-\mathbf{2 g}$ were evaluated for their in vitro antibacterial activity against Staphylococcus aureus (ATCC-25923), Escherichia coli (ATCC-25922), Enterococcus feacalis, Pseudomonas aeruginosa (ATCC27853), Klebsiella pneumoniae (recultured) by agar diffusion method [15,16] was performed using Mueller-Hinton agar (Hi-Media) medium.

Each compound was tested at a concentration of $100 \mu \mathrm{g} / \mathrm{mL}$ in DMSO. Ciprofloxacin was used as the standard. The zone of inhibition was measured after $24 \mathrm{~h}$ incubation at $37^{\circ} \mathrm{C}$ (Table 2). The minimum inhibitory concentration (MIC) was considered to be the lowest concentration that completely inhibited growth on agar plates.

\section{In vitro antifungal screening}

Compounds $\mathbf{1 a}-\mathbf{1 g}$ and $\mathbf{2} \mathbf{a}-\mathbf{2 g}$ were evaluated for their in vitro antifungal activity Aspergillus niger, Candia albicans, Aspergillus fumigatus, Cryptococcus neoformans and Microsporum audouinii (recultured) using an agar diffusion method $[17,18]$ with Sabouraud's dextrose agar (Hi-Media). Each compound was tested at a concentration of $100 \mu \mathrm{g} / \mathrm{mL}$ in DMSO. Clotrimazole was used as the standard. The zone of inhibition was measured incubated at 37 ${ }^{\circ} \mathrm{C}$ for $24 \mathrm{~h}$ and MICs were determined.

\section{Evaluation of larvicidal activity}

The assessment of larvicidal activity of synthesized test compounds $\mathbf{1 a - 1 g}$ and $\mathbf{2 a - 2 g}$ was tested against the urban mosquitoes Culex. Quinquefasciatus using standard bioassay protocol [19]. Egg rafts of mosquito were obtained from a drainage system. The eggs were reared under standard insectary conditions at ambient temperature $\left(29 \pm 3{ }^{\circ} \mathrm{C}\right)$ and relative humidity ( $80 \pm 5 \%), 12: 12$ light: dark photoperiod and fed with ground shrimp feed daily.

Larval development was monitored for 7 days. The second and third stage larvae were collected at the tip of a pasture pipette and placed in cotton bud to remove excess water and transferred gently to the test vial (10/vial) by tapping. The larval mortality was observed using various concentrations of synthesized compounds (10, 20,30, and $40 \mu \mathrm{g} / \mathrm{mL}$ ).

\section{Assessment of nematicidal activity}

For the determination of nematicidal activity, juveniles of Meloidogyne javanica were used as test organism [19]. Assay system was prepared with $2 \mathrm{ml}$ Milli $Q$ water containing different concentrations $(10,20,30$ and $40 \mu \mathrm{g} / \mathrm{mL})$ of synthesized test compounds 1-6 in glass tubes.

Ten juveniles of $M$. javanica were transferred in test, positive (with $2 \%$ methanol) and negative (without vehicle) control tubes. Mortality was observed under a zoom stereomicroscope after $24 \mathrm{~h}$ of exposure.

\section{Statistical analysis}

The mean of the results was calculated based on at least 3 independent evaluations and the standard deviations (SD) were also calculated using Microsoft Excel. All $\mathrm{LD}_{50}$ values were calculated from the corresponding sigmoidal dose-response curve according to best fit shapes based on at least five reaction points using the Microsoft Office Excel 2007 software (Microsoft, Redmond, WA, USA).

\section{RESULTS}

\section{Chemistry}

\section{2-[1 H-Imidazol-1-yl (phenyl) methyl] hydrazinecarbothioamide (1a)}

$\mathrm{IR}\left(\mathrm{KBr}, \mathrm{cm}^{-1}\right): \quad 3292.26\left(\mathrm{NH}_{2}\right), \quad$ 2916.17(NH), 1436.87(C=S), 811.98(Ar-H); 460(CH) ${ }^{1} \mathrm{HNMR}$ (DMSO-d $6,300 \mathrm{MHz}): 9.53\left(\mathrm{~s}, 2 \mathrm{H}, \mathrm{NH}_{2}\right), 7.83(\mathrm{~s}$, $1 \mathrm{H}, \mathrm{CH}), 7.26-7.65(\mathrm{~m}, 5 \mathrm{H}$, Phenyl), 6.77(s, $1 \mathrm{H}$, Imidazole-CH), $6.84(\mathrm{~s}, 1 \mathrm{H}, \mathrm{CH}), 2.2(1 \mathrm{H}, \mathrm{NH})$, $2.0(\mathrm{~s}, 1 \mathrm{H}, \mathrm{NH}){ }^{13} \mathrm{C}$ NMR (DMSO-d $\left.6,300 \mathrm{MHz}\right)$; 182.7 (C=S), 128.7, 126.6, 128.1, 132.5 (Pheny), 128.6 (Imidazole ring $\mathrm{CH}=\mathrm{CH}), 137.8(\mathrm{HC}=\mathrm{N})$, $75.3(\mathrm{CH})$. El-MS, m/z (Relative intensity \%): [247.28 $\left.{ }^{+}, 42 \%\right]$.

\section{2-((4-Chlorophenyl) (1H-imidazol-1-yl) methyl) hydrazinecarbothioamide (1b)}

$\mathrm{IR}\left(\mathrm{KBr}, \mathrm{Cm}^{-1}\right): \quad$ 3291.44( $\left(\mathrm{NH}_{2}\right), \quad$ 2912.34(NH), 1440.88(C=S), 837(C-Cl), 819.18(Ar-H), 456(CH); ${ }^{1} \mathrm{H}$ NMR (DMSO-d 6 , $\left.300 \mathrm{MHz}\right): 9.43(\mathrm{~s}$, $\left.2 \mathrm{H}, \mathrm{NH}_{2}\right), 7.62(\mathrm{~s}, 1 \mathrm{H}, \mathrm{CH}), 7.36-7.16(\mathrm{tt}, 4 \mathrm{H}$, Phenyl), 6.55(s, 1H, Imidazole-CH), 6.72(s, 1H, $\mathrm{CH}), \quad 2.3(1 \mathrm{H}, \mathrm{NH}), \quad 2.1(\mathrm{~s}, 1 \mathrm{H}, \mathrm{NH}) ; \quad{ }^{13} \mathrm{C}$ NMR(DMSO- $\left.\mathrm{d}_{6}, 300 \mathrm{MHz}\right) ; 1810.7$ (C=S), 136.6, 132.3, 128.6, 128.3 (Ph-Cl), 128.0 (Imidazole ring $\mathrm{CH}=\mathrm{CH}), 138.1(\mathrm{HC}=\mathrm{N}), 76.8(\mathrm{CH})$. El-MS, $\mathrm{m} / \mathrm{z}$ (Relative intensity \%): [281.76 $\left.{ }^{+}, 37 \%\right]$. 
2-((4-Hydroxyphenyl) (1H-imidazol-1-yl) methyl) hydrazinecarbothioamide (1c)

IR $\left(\mathrm{KBr}, \mathrm{cm}^{-1}\right): \quad 3290.21\left(\mathrm{NH}_{2}\right), \quad 2914.16(\mathrm{NH})$, 1435.83(C=S), 809.97(Ar-H), 458(CH); ${ }^{1} \mathrm{HNMR}$ $\left(\mathrm{DMSO}_{\mathrm{d}}, 300 \mathrm{MHz}\right): \quad 9.52\left(\mathrm{~s}, 2 \mathrm{H}, \mathrm{NH}_{2}\right), \quad 9.47$ $(1 \mathrm{H}, \mathrm{s}, \mathrm{C}-\mathrm{OH}), 9.43$ (s, $1 \mathrm{H}, 9.43), 7.80(\mathrm{~s}, 1 \mathrm{H}$, $\mathrm{CH}), 6.63-7.08$ (tt, 4H, Phenyl), 6.73 (s, 1H, Imidazole-CH), $6.78(\mathrm{~s}, 1 \mathrm{H}, \mathrm{CH}), 2.4(1 \mathrm{H}, \mathrm{NH})$, $2.1(\mathrm{~s}, 1 \mathrm{H}, \mathrm{NH}) ;{ }^{13} \mathrm{C}$ NMR(DMSO-d $\left.6,300 \mathrm{MHz}\right)$; $1821.4(\mathrm{C}=\mathrm{S}), 115.7,128.3,131.2(\mathrm{Ph}-\mathrm{OH})$, 127.9 (Imidazole ring $\mathrm{CH}=\mathrm{CH}), 138.2(\mathrm{HC}=\mathrm{N})$, 74.2(CH). El-MS, $\mathrm{m} / \mathrm{z}$ (Relative intensity \%): [263.31 $\left.{ }^{+}, 26 \%\right]$.

\section{2-((1H-Imidazol-1-yl) (4-nitrophenyl) methyl) hydrazinecarbothioamide (1d)}

$\mathrm{IR}\left(\mathrm{KBr}, \mathrm{cm}^{-1}\right): \quad 3294.29 \quad\left(\mathrm{NH}_{2}\right), \quad 2915.15 \quad(\mathrm{NH})$, $1435.88(\mathrm{C}=\mathrm{S}), 814.90(\mathrm{Ar}-\mathrm{H}), 1536$ (C-NO2), $458.92(\mathrm{CH}) ;{ }^{1} \mathrm{H}$ NMR (DMSO-d $\left.6,300 \mathrm{MHz}\right): 9.51$ (s, 2H, $\left.\mathrm{NH}_{2}\right), 7.80(\mathrm{~s}, 1 \mathrm{H}, \mathrm{CH}), 7.22-7.64(\mathrm{~m}, 5 \mathrm{H}$, Phenyl), 6.77 (s, 1H, Imidazole-CH), 6.84 (s, 1H, $\mathrm{CH}), 2.4(\mathrm{~s}, 1 \mathrm{H}, \mathrm{NH}), 2.1(\mathrm{~s}, 1 \mathrm{H}, \mathrm{NH}) ;{ }^{13} \mathrm{C}$ NMR (DMSO-d, $300 \mathrm{MHz}) ; 181.8$ (C=S), 155.2, 131.6, 128.3, $127.9\left(\mathrm{Ph}-\mathrm{NO}_{2}\right), 128.2$ (Imidazole ring $\mathrm{CH}=\mathrm{CH}), 136.3(\mathrm{HC}=\mathrm{N}), 74.8(\mathrm{CH}) ; \mathrm{El}-\mathrm{MS}, \mathrm{m} / \mathrm{z}$ (Relative intensity \%): [292.31 ${ }^{+}, 36 \%$ ].

\section{2-((1H-imidazol-1-yl) (4-methoxyphenyl) methyl) hydrazinecarbothioamide (1e)}

IR $\left(\mathrm{KBr}, \mathrm{cm}^{-1}\right): 3288.20\left(\mathrm{NH}_{2}\right), 2912.14(\mathrm{NH})$, 1432.80 (C=S), $812.95(\mathrm{Ar}-\mathrm{H}), 458.67(\mathrm{CH}) ;{ }^{1} \mathrm{H}$ NMR (DMSO-d 6 , 300MHz): $9.51\left(\mathrm{~s}, 2 \mathrm{H}, \mathrm{NH}_{2}\right.$ ), $7.88(\mathrm{~s}, 1 \mathrm{H}, \mathrm{CH}), \quad 6.88-7.10(\mathrm{tt}, 4 \mathrm{H}, \mathrm{Ph}-$ $\left.\mathrm{OCH}_{3}\right), 6.68$ (s, 1H, Imidazole- $\left.\mathrm{CH}\right), 6.80$ (s, 1H, $\mathrm{CH}), 3.84$ (s, 3H, -OCH3), 2.1 (s, 1H, NH), 2.0 (s, $1 \mathrm{H}, \mathrm{NH}) ;{ }^{13} \mathrm{C}$ NMR (DMSO-d, $300 \mathrm{MHz}$ ): $1818.6(\mathrm{C}=\mathrm{S}), 158.6,130.9,114.0,126.0(\mathrm{Ph}-$ $\mathrm{OH}), 128.1$ (Imidazole ring $\mathrm{CH}=\mathrm{CH}), 55.7(\mathrm{Ph}-$ $\mathrm{OCH} 3), 134.2$ (HC=N), 74. 8(CH); El-MS, m/z (Relative intensity \%): [277.34 ${ }^{+}, 28 \%$ ].

\section{2-((4-(dimethylamino) phenyl) (1H-imidazol-1-} yl) methyl) hydrazinecarbothioamide (1f)

IR $\left(\mathrm{KBr}, \mathrm{cm}^{-1}\right): 3282.16\left(\mathrm{NH}_{2}\right), 2912.12(\mathrm{NH})$, 1438.82 (C=S), $808.92(\mathrm{Ar}-\mathrm{H}), 460(\mathrm{CH}) ;{ }^{1} \mathrm{H}$ NMR (DMSO-d $6,300 \mathrm{MHz}): 9.53\left(\mathrm{~s}, 2 \mathrm{H}, \mathrm{NH}_{2}\right)$, 7.83 (s, 1H, CH), 7.02 - 6.64 (tt, 4H, Ph $\left.\mathrm{N}\left(\mathrm{CH}_{3}\right)_{2}\right), 6.70(\mathrm{~s}, 1 \mathrm{H}$, Imidazole- $\mathrm{CH}), 3.12(\mathrm{~s}$, $\left.6 \mathrm{H},-\mathrm{N}\left(\mathrm{CH}_{3}\right)_{2}\right), 6.84(\mathrm{~s}, 1 \mathrm{H}, \mathrm{CH}), 2.2(1 \mathrm{H}, \mathrm{NH})$, 2.0 (s, 1H, NH); ${ }^{13} \mathrm{C}$ NMR (DMSO-d, $\left.300 M h z\right)$ : 180.6 (C=S), 148.3, 128.1, 127.2, 112.8, 40.2 $\left(\mathrm{Ph}-\mathrm{N}\left(\mathrm{CH}_{3}\right)_{2}\right), 126.2$ (Imidazole ring $\mathrm{CH}=\mathrm{CH}$ ), $136.8(\mathrm{HC}=\mathrm{N}), 40.8\left(-\mathrm{N}\left(\mathrm{CH}_{3}\right)_{2}\right), 74.0(\mathrm{CH})$; ElMS, m/z (Relative intensity \%): [290.38 $\left.{ }^{+}, 37 \%\right]$.

\section{2-((1H-Imidazol-1-yl) (p-tolyl) methyl) hydrazinecarbothioamide (1g)}

$\mathrm{IR}\left(\mathrm{KBr}, \mathrm{cm}^{-1}\right): \quad 3280.22\left(\mathrm{NH}_{2}\right), 2914.13(\mathrm{NH})$, $1430.82(\mathrm{C}=\mathrm{S}), 814.96(\mathrm{Ar}-\mathrm{H}), 454.89(\mathrm{CH}) ;{ }^{1} \mathrm{H}$ NMR (DMSO-d, $300 \mathrm{MHz}): 9.50\left(\mathrm{~s}, 2 \mathrm{H}, \mathrm{NH}_{2}\right.$ ), $7.82(\mathrm{~s}, 1 \mathrm{H}, \mathrm{CH}), 7.11$ - $7.14(\mathrm{tt}, 4 \mathrm{H}, \mathrm{Ph}-$ $\left.\mathrm{CH}_{3}\right), 6.56(\mathrm{~s}, 1 \mathrm{H}$, Imidazole- $\mathrm{CH}), 6.82(\mathrm{~s}, 1 \mathrm{H}$, $\mathrm{CH}), 2.42(\mathrm{~s}, 1 \mathrm{H}, \mathrm{NH}), 2.33\left(\mathrm{~s}, 3 \mathrm{H},-\mathrm{CH}_{3}\right), 2.21$ (s, $1 \mathrm{H}, \mathrm{NH}) ;{ }^{13} \mathrm{C}$ NMR (DMSO-d, $\left.300 \mathrm{MHz}\right)$; 184.5 (C=S), 135.9, 135.4, 128.5, 126.8 (Ph $\mathrm{CH}_{3}$ ), 128.5 (Imidazole ring $\mathrm{CH}=\mathrm{CH}$ ), 137.2 $(\mathrm{HC}=\mathrm{N}), 74.6 \quad(\mathrm{CH}) ; \mathrm{El}-\mathrm{MS}, \mathrm{m} / \mathrm{z}$ (Relative intensity \%): [261.34 $\left.{ }^{+}, 22 \%\right]$.

\section{2-[1H-Imidazol-1-yl (phenyl) methyl] hydrazinecarboxamide (2a)}

$\mathrm{IR}\left(\mathrm{KBr}, \mathrm{cm}^{-1}\right)$ : $3287.20\left(\mathrm{NH}_{2}\right), 2932.97(\mathrm{NH})$, $1623.21(\mathrm{C}=\mathrm{O}), 819.78(\mathrm{Ar}-\mathrm{H}), 456.98(\mathrm{CH}){ }^{1} \mathrm{H}$ NMR (DMSO d $\left.\mathrm{d}_{6}, 300 \mathrm{MHz}\right): 9.50\left(\mathrm{~s}, 2 \mathrm{H}, \mathrm{NH}_{2}\right.$ ), 7.86 (s, 1H, CH), 7.26-7.65 (m, 5H, Phenyl), 6.89 (s, 1H, Imidazole- $\mathrm{H}), 6.76(\mathrm{~s}, 1 \mathrm{H}, \mathrm{CH}), 2.21$ $(1 \mathrm{H}, \mathrm{NH}), 2.09(\mathrm{~s}, 1 \mathrm{H}, \mathrm{NH}){ }^{13} \mathrm{C}$ NMR $\left(\mathrm{DMSOd}_{6}\right.$, 300MHz); 157.9 (C=O), 126.1, 126.8, 128.5, 138.9 (Phenyl), 128.1 (Imidazole ring $\mathrm{CH}=\mathrm{CH}$ ), $137.1(\mathrm{HC}=\mathrm{N}), 75.9(\mathrm{CH})$. El-MS, m/z (Relative intensity \%): [231.28 $\left.{ }^{+}, 51 \%\right]$.

2-((4-Chlorophenyl) (1H-imidazol-1-yl) methyl) hydrazinecarboxamide (2b)

IR $\left(\mathrm{KBr}, \mathrm{cm}^{-1}\right): 3281.18\left(\mathrm{NH}_{2}\right), 2930.92(\mathrm{NH})$, $1621.18(\mathrm{C}=\mathrm{O}), 837(\mathrm{C}-\mathrm{Cl}), 818.71(\mathrm{Ar}-\mathrm{H}), 455.91$ $(\mathrm{CH}) ;{ }^{1} \mathrm{H}$ NMR (DMSO d, $\left.300 \mathrm{MHz}\right): 9.52$ (s, $2 \mathrm{H}$, $\left.\mathrm{NH}_{2}\right), 7.82$ (s, 1H, CH), $7.36-7.16$ (tt, $4 \mathrm{H}$, Phenyl), 6.86 (s, 1H, Imidazole- $\mathrm{CH}), 6.74$ (s, 1H, $\mathrm{CH}), 2.20(1 \mathrm{H}, \mathrm{NH}), 2.12(\mathrm{~s}, 1 \mathrm{H}, \mathrm{NH}) ;{ }^{13} \mathrm{C}$ NMR $\left(\mathrm{DMSOd}_{6}, 300 \mathrm{MHz}\right) ; 157.2(\mathrm{C}=\mathrm{O}), 136.6,132.3$, 128.6, 128.3 (Ph-Cl) 128.4 (Imidazole ring $\mathrm{CH}=\mathrm{CH}), 137.6(\mathrm{HC}=\mathrm{N}), 76.5(\mathrm{CH}) ; \mathrm{El}-\mathrm{MS}, \mathrm{m} / \mathrm{z}$ (Relative intensity \%): [265.70 $+38 \%]$.

\section{2-((4-Hydroxyphenyl) (1H-imidazol-1-yl) methyl) hydrazinecarboxamide (2c)}

IR $\left(\mathrm{KBr}, \mathrm{cm}^{-1}\right): 3280.24\left(\mathrm{NH}_{2}\right), 2930.94(\mathrm{NH})$, $1624.23(\mathrm{C}=\mathrm{O}), 816.76(\mathrm{Ar}-\mathrm{H}), 1472(\mathrm{C}-\mathrm{OH})$, 457.91(CH); ${ }^{1} \mathrm{H}$ NMR (DMSO d, $300 \mathrm{MHz}$ ): 9.46 (s, 2H, $\left.\mathrm{NH}_{2}\right), 9.41(1 \mathrm{H}, \mathrm{s}, \mathrm{OH}), 7.84(\mathrm{~s}, 1 \mathrm{H}, \mathrm{CH})$, 6.63 - 7.08 (tt, 4H, Phenyl), 6.86 (s, 1H, Imidazole- $\mathrm{CH}), 6.74$ (s, 1H, CH), $2.19(1 \mathrm{H}, \mathrm{NH})$, $2.12(\mathrm{~s}, 1 \mathrm{H}, \mathrm{NH}) ;{ }^{13} \mathrm{C}$ NMR $\left(\mathrm{DMSOd}_{6}, 300 \mathrm{MHz}\right)$; 155.6 (C=O), 125.2, 126.6, 128.2, 138.4 (Phenyl), 128.4 (Imidazole ring $\mathrm{CH}=\mathrm{CH}), 136.5$ $(\mathrm{HC}=\mathrm{N}), 115.7,128.3,131.2(\mathrm{Ph}-\mathrm{OH}), 75.2$ $(\mathrm{CH}) ; \quad \mathrm{El}-\mathrm{MS}, \mathrm{m} / \mathrm{z}$ (Relative intensity \%): $\left[247.25^{+}, 33 \%\right]$. 
2-((1H-Imidazol-1-yl) (4-nitrophenyl) methyl) hydrazinecarboxamide (2d)

$\mathrm{IR}\left(\mathrm{KBr}, \mathrm{cm}^{-1}\right): 3278.10\left(\mathrm{NH}_{2}\right), 2930.91(\mathrm{NH})$, $1622.18(\mathrm{C}=\mathrm{O}), 813.18(\mathrm{Ar}-\mathrm{H}), 1530(\mathrm{C}-\mathrm{NO} 2)$, 455.91(CH); ${ }^{1} \mathrm{H}$ NMR (DMSO d $6,300 \mathrm{MHz}$ ): 9.46 (s, $\left.2 \mathrm{H}, \mathrm{NH}_{2}\right), 7.84(\mathrm{~s}, 1 \mathrm{H}, \mathrm{CH}), 7.26-7.65(\mathrm{~m}, 5 \mathrm{H}$, Phenyl), 6.88 (s, 1H, Imidazole-CH), 6.74 (s, 1H, $\mathrm{CH}), 2.18(1 \mathrm{H}, \mathrm{NH}), 2.12(\mathrm{~s}, 1 \mathrm{H}, \mathrm{NH}) ;{ }^{13} \mathrm{C} \mathrm{NMR}$ $\left(\mathrm{DMSOd}_{6}, 300 \mathrm{MHz}\right) ; 156.8(\mathrm{C}=\mathrm{O}), 155.2,131.6$, 128.3, $127.9\left(\mathrm{Ph}-\mathrm{NO}_{2}\right), 128.6$ (Imidazole ring $\mathrm{CH}=\mathrm{CH}), 136.4(\mathrm{HC}=\mathrm{N}), 75.2(\mathrm{CH}) . \quad \mathrm{El}-\mathrm{MS}, \mathrm{m} / \mathrm{z}$ (Relative intensity \%): [276.25, $30 \%]$.

\section{2-((1H-Imidazol-1-yl) (4-methoxyphenyl) methyl) hydrazinecarboxamide (2e)}

IR $\left(\mathrm{KBr}, \mathrm{cm}^{-1}\right): 3276.10\left(\mathrm{NH}_{2}\right), 2936.87(\mathrm{NH})$, 1628.22 (C=O), $816.72(\mathrm{Ar}-\mathrm{H}), 456.94(\mathrm{CH}){ }^{1} \mathrm{H}$ NMR (DMSO-d $\left.\mathrm{d}_{6}, 300 \mathrm{MHz}\right): 9.54\left(\mathrm{~s}, 2 \mathrm{H}, \mathrm{NH}_{2}\right.$ ), $7.85(\mathrm{~s}, 1 \mathrm{H}, \mathrm{CH}),\left(\mathrm{tt}, 4 \mathrm{H}, \mathrm{Ph}-\mathrm{OCH}_{3}\right), 6.86(\mathrm{~s}$, $1 \mathrm{H}$, Imidazole-CH), $6.74(\mathrm{~s}, 1 \mathrm{H}, \mathrm{CH}), 3.81(3 \mathrm{H}, s$, $-\mathrm{OCH} 3), 2.18(1 \mathrm{H}, \mathrm{NH}), 2.10(\mathrm{~s}, 1 \mathrm{H}, \mathrm{NH}) ;{ }^{13} \mathrm{C}$ NMR (DMSO-d $\left.\mathrm{d}_{6}, 300 \mathrm{MHz}\right) ; 158.6(\mathrm{C}=\mathrm{O}), 158.6$, 130.9, 114.0, $126.0(\mathrm{Ph}-\mathrm{OH}), 128.2$ (Imidazole ring $\mathrm{CH}=\mathrm{CH}), 137.3(\mathrm{HC}=\mathrm{N}), 55.9(-\mathrm{OCH} 3) ; 74.8$ $(\mathrm{CH}) ; \quad \mathrm{El}-\mathrm{MS}, \mathrm{m} / \mathrm{z}$ (Relative intensity \%): [261.28, $54 \%]$.

2-((4-(Dimethylamino) phenyl) (1H-imidazol-1yl) methyl) hydrazinecarboxamide (2f)

$\mathrm{IR}\left(\mathrm{KBr}, \mathrm{cm}^{-1}\right): 3281.21\left(\mathrm{NH}_{2}\right), 2929.91(\mathrm{NH})$, 1627.27 (C=O), $817.71(\mathrm{Ar}-\mathrm{H}), 453.96(\mathrm{CH}) ;{ }^{1} \mathrm{H}$ NMR (DMSO-d $\left.\mathrm{d}_{6}, 300 \mathrm{MHz}\right): 9.47\left(\mathrm{~s}, 2 \mathrm{H}, \mathrm{NH}_{2}\right.$ ), $7.81(\mathrm{~s}, 1 \mathrm{H}, \mathrm{CH}),\left(\mathrm{tt}, 4 \mathrm{H}, \mathrm{Ph}-\mathrm{N}\left(\mathrm{CH}_{3}\right)_{2}\right), 6.82(\mathrm{~s}$, $1 \mathrm{H}$, Imidazole-CH), $6.72(\mathrm{~s}, 1 \mathrm{H}, \mathrm{CH}), 3.06(1 \mathrm{H}, s$, $-\mathrm{N}(\mathrm{CH} 3) 2), 2.27(1 \mathrm{H}, \mathrm{NH}), 2.12(\mathrm{~s}, 1 \mathrm{H}, \mathrm{NH}) ;{ }^{13} \mathrm{C}$ NMR (DMSO-d 6 , 300MHz); $156.2(\mathrm{C}=\mathrm{O}), 148.3$, 128.1, 127.2, 112.8, 40.2 (Ph - N $\left.\left(\mathrm{CH}_{3}\right)_{2}\right)$, 128.6 (Imidazole ring $\mathrm{CH}=\mathrm{CH}), \quad 136.5(\mathrm{HC}=\mathrm{N})$, $40.8(\mathrm{~N}(\mathrm{CH} 3) 2), 76.2(\mathrm{CH})$. El-MS, m/z (Relative intensity \%): [274.32 $\left.{ }^{+}, 23 \%\right]$.

\section{2-((1H-Imidazol-1-yl)(p-tolyl) methyl) hydrazinecarboxamide (2g)}

IR $\left(\mathrm{KBr}, \mathrm{cm}^{-1}\right): \quad 3266.21\left(\mathrm{NH}_{2}\right), \quad 2930.70(\mathrm{NH})$, 1622.19(C=O), 818.18(Ar-H), $455.18(\mathrm{CH}) ;{ }^{1} \mathrm{H}$ NMR (DMSO- $\left.\mathrm{d}_{6}, 300 \mathrm{MHz}\right): 9.52\left(\mathrm{~s}, 2 \mathrm{H}, \mathrm{NH}_{2}\right.$ ), 7.81(s, 1H, CH), 7.26-7.65(tt, 4H, Phenyl), 6.87 (s, 1H, Imidazole- $\mathrm{CH}), 6.75(\mathrm{~s}, 1 \mathrm{H}, \mathrm{CH}), 2.34(\mathrm{~s}$, $\left.3 \mathrm{H}, \mathrm{CH}_{3}\right), 2.26(1 \mathrm{H}, \mathrm{NH}), 2.10(\mathrm{~s}, 1 \mathrm{H}, \mathrm{NH}) ;{ }^{13} \mathrm{C}$ NMR (DMSO-d $6,300 \mathrm{MHz}) ; 155.6(\mathrm{C}=\mathrm{O}), 135.9$, 135.4, 128.5, $126.8\left(\mathrm{Ph}-\mathrm{CH}_{3}\right), 128.8$ (Imidazole ring $\mathrm{CH}=\mathrm{CH}), 136.9(\mathrm{HC}=\mathrm{N}), 75.1(\mathrm{CH})$. El-MS, $\mathrm{m} / \mathrm{z}$ (Relative intensity \%): [245.28 $\left.{ }^{+}, 56 \%\right]$.

\section{Antibacterial activity}

Compounds $\mathbf{1 a - g}$ and $\mathbf{2 a}-\mathbf{2 g}$ were evaluated for in vitro antibacterial activity against Staphylococcus aureus, Escherichia coli, Enterococcus. faecalis. Pseudomonas aeruginosa and Klebsiella pneumoniae using conventional agar dilution procedures. Ciprofloxacin was used as a positive control. Inhibition zones were measured and compared against those of controls. The bacterial zones of inhibition are given in Table 2.

Compared with ciprofloxacin, compound $\mathbf{1 b}$ was highly active against $S$. aureus, and $\mathbf{1 g}$ exhibited an equivalent activity $(0.5 \mu \mathrm{g} / \mathrm{mL})$ against $E$. faecalis and greater activity $(0.25 \mu \mathrm{g} / \mathrm{mL})$ against $P$. aeruginosa. Compound $2 \mathrm{e}$ was highly active against $K$. pneumonia and $\mathbf{2} \mathbf{f}$ showed equivalent activities $(0.5 \mu \mathrm{g} / \mathrm{mL})$ against $E$. coli and $E$. feacalis. Compound $\mathbf{2 g}$ was highly active (0.5 $\mu \mathrm{g} / \mathrm{mL}$ ) against $P$. aeruginosa. The MIC values are summarised in Table 3.

\section{Antifungal activity}

Compounds 1a-g and 2a-g were evaluated in terms of their in vitro antifungal activity against Aspergillus niger, Candida albicans, Aspergillus fumigatus, Cryptococcus neoformans (recultured), and Microsporum audouinii using an agar diffusion method. The fungal activity of each compound was compared with that of clotrimazole as positive control. The fungal zones of inhibition are given in Table 2. Compounds $\mathbf{2 d}$ and $\mathbf{2 g}(0.25 \mu \mathrm{g} / \mathrm{mL})$ were highly active against C. albicans. Compound $\mathbf{2 d}$ was equipotent active $(1 \mu \mathrm{g} / \mathrm{mL})$ against $M$. audouinii compared with clotrimazole (MIC, $2 \mu \mathrm{g} / \mathrm{ml}$ ).

\section{Larvicidal activity}

The larvicidal activity of the test compounds is listed in Table 4. Larvicidal activity was determined for compounds $\mathbf{1 a - g}$ and $\mathbf{2 a - g}$ by exposing second instar larvae for $24 \mathrm{~h}$ at room temperature. With the exception of $\mathbf{1 g}$, the compounds exhibited moderate larvicidal activity against mosquito. Compounds 1a, 1b, 1d, 1f, 2a, $\mathbf{2 b}, \mathbf{2 c}, \mathbf{2 d}, \mathbf{2 f}$ and $\mathbf{2 g}$ ) yielded $100 \%$ mortality at $40 \mu \mathrm{g} / \mathrm{mL}$. Compound $1 \mathrm{~g}$ was particularly toxic with an $\mathrm{LD}_{50}$ of $9.5 \mu \mathrm{g} / \mathrm{mL}$.

\section{Nematicidal activity}

Compounds 1a-g and 2a-g were alao evaluated in terms of their in vitro nematicidal activity against Meloidogyne javanica at various aqueous concentrations. Compound $\mathbf{1 g}$ was the most effective nematicide as evidenced by its $L D_{50}$ of 
$8.9 \mu \mathrm{g} / \mathrm{mL}$. Compounds (1d, 1e, $\mathbf{1 f}, \mathbf{1 g}, \mathbf{2 d}$ and mortality at $30 \mu \mathrm{g} / \mathrm{mL}$. $\mathrm{LD}_{50}$ values are reported 2e) were more potent then compounds $(\mathbf{1 a}, \mathbf{1 b}$, in Table 5 .

2a, 2b, 2c, 2f, and $\mathbf{2 g}$ ), which exhibited $100 \%$

Table 1: Physicochemical data of the compounds (1a-g) and (2a-g)

\begin{tabular}{|c|c|c|c|c|c|c|c|c|}
\hline \multirow[t]{2}{*}{$\begin{array}{c}\text { Compound } \\
\text { no. }\end{array}$} & \multirow[t]{2}{*}{$\mathbf{R}$} & \multirow[t]{2}{*}{ M.W } & \multirow[t]{2}{*}{ M.F } & \multirow[t]{2}{*}{ M.P(C) } & \multicolumn{4}{|c|}{$\begin{array}{l}\text { Elemental analysis } \\
\text { (Calculated \& found) }\end{array}$} \\
\hline & & & & & C & $\mathrm{H}$ & $\mathbf{N}$ & $\mathbf{S}$ \\
\hline $1^{\mathrm{a}}$ & $-\mathrm{H}$ & 247.31 & $\mathrm{C}_{11} \mathrm{H}_{13} \mathrm{~N}_{5} \mathrm{~S}$ & 130 & $\begin{array}{c}53.42 \\
(53.40)\end{array}$ & $\begin{array}{c}5.30 \\
(5.27)\end{array}$ & $\begin{array}{l}28.32 \\
(28.30)\end{array}$ & $\begin{array}{c}12.97 \\
(12.96)\end{array}$ \\
\hline 1b & $-\mathrm{Cl}$ & 281.76 & $\mathrm{C}_{11} \mathrm{H}_{12} \mathrm{Cl} \mathrm{N} \mathrm{N}_{5} \mathrm{~S}$ & 140 & $\begin{array}{c}46.89 \\
(46.87)\end{array}$ & $\begin{array}{c}4.29 \\
(4.28)\end{array}$ & $\begin{array}{c}24.86 \\
(24.84)\end{array}$ & $\begin{array}{c}11.38 \\
(11.36)\end{array}$ \\
\hline 1c & $-\mathrm{OH}$ & 263.31 & $\mathrm{C}_{11} \mathrm{H}_{13} \mathrm{~N}_{5} \mathrm{OS}$ & 137 & $\begin{array}{c}50.17 \\
(50.15)\end{array}$ & $\begin{array}{l}4.98 \\
(4.97)\end{array}$ & $\begin{array}{l}26.60 \\
(26.63)\end{array}$ & $\begin{array}{c}12.18 \\
(12.17)\end{array}$ \\
\hline 1d & $-\mathrm{NO}_{2}$ & 292.31 & $\mathrm{C}_{11} \mathrm{H}_{12} \mathrm{~N}_{6} \mathrm{O}_{2} \mathrm{~S}$ & 143 & $\begin{array}{c}45.20 \\
(45.22)\end{array}$ & $\begin{array}{c}4.14 \\
(4.10)\end{array}$ & $\begin{array}{c}28.75 \\
(28.72)\end{array}$ & $\begin{array}{c}10.97 \\
(10.98)\end{array}$ \\
\hline $1 e$ & $-\mathrm{OCH}_{3}$ & 277.34 & $\mathrm{C}_{12} \mathrm{H}_{15} \mathrm{~N}_{5} \mathrm{OS}$ & 147 & $\begin{array}{c}51.97 \\
(51.99)\end{array}$ & $\begin{array}{l}5.45 \\
(5.41)\end{array}$ & $\begin{array}{l}25.25 \\
(25.23)\end{array}$ & $\begin{array}{c}11.56 \\
(11.55)\end{array}$ \\
\hline $1 f$ & $-\mathrm{N}\left(\mathrm{CH}_{3}\right)_{2}$ & 290.38 & $\mathrm{C}_{13} \mathrm{H}_{18} \mathrm{~N}_{6} \mathrm{~S}$ & 164 & $\begin{array}{c}53.77 \\
(53.78)\end{array}$ & $\begin{array}{c}6.25 \\
(6.24)\end{array}$ & $\begin{array}{c}28.94 \\
(28.90)\end{array}$ & $\begin{array}{c}11.04 \\
(11.08)\end{array}$ \\
\hline $1 \mathrm{~g}$ & $-\mathrm{CH}_{3}$ & 261.34 & $\mathrm{C}_{12} \mathrm{H}_{15} \mathrm{~N}_{5} \mathrm{~S}$ & 161 & $\begin{array}{c}55.15 \\
(55.14)\end{array}$ & $\begin{array}{c}5.79 \\
(5.78)\end{array}$ & $\begin{array}{l}26.80 \\
(26.83)\end{array}$ & $\begin{array}{c}12.27 \\
(12.29)\end{array}$ \\
\hline $\mathbf{2}^{\mathrm{a}}$ & $-H$ & 231.25 & $\mathrm{C}_{11} \mathrm{H}_{13} \mathrm{~N}_{5} \mathrm{O}$ & 110 & $\begin{array}{c}57.13 \\
(57.10)\end{array}$ & $\begin{array}{c}5.67 \\
(5.66)\end{array}$ & $\begin{array}{c}30.28 \\
(30.26)\end{array}$ & - \\
\hline $2 b$ & $-\mathrm{Cl}$ & 265.70 & $\mathrm{C}_{11} \mathrm{H}_{12} \mathrm{CIN}_{5} \mathrm{O}$ & 122 & $\begin{array}{c}49.72 \\
(49.70)\end{array}$ & $\begin{array}{l}4.55 \\
(4.53)\end{array}$ & $\begin{array}{l}26.36 \\
(26.33)\end{array}$ & - \\
\hline $2 c$ & $-\mathrm{OH}$ & 247.25 & $\mathrm{C}_{11} \mathrm{H}_{13} \mathrm{~N}_{5} \mathrm{O}_{2}$ & 137 & $\begin{array}{c}53.43 \\
(53.40)\end{array}$ & $\begin{array}{l}5.30 \\
(5.31)\end{array}$ & $\begin{array}{l}28.32 \\
(28.30)\end{array}$ & - \\
\hline $2 d$ & $-\mathrm{NO}_{2}$ & 276.25 & $\mathrm{C}_{11} \mathrm{H}_{12} \mathrm{~N}_{6} \mathrm{O}_{3}$ & 146 & $\begin{array}{c}47.83 \\
(47.80)\end{array}$ & $\begin{array}{l}4.38 \\
(4.34)\end{array}$ & $\begin{array}{c}30.42 \\
(30.41)\end{array}$ & - \\
\hline $2 e$ & $-\mathrm{OCH}_{3}$ & 261.28 & $\mathrm{C}_{12} \mathrm{H}_{15} \mathrm{~N}_{5} \mathrm{O}_{2}$ & 137 & $\begin{array}{c}55.16 \\
(55.13)\end{array}$ & $\begin{array}{c}5.79 \\
(5.78)\end{array}$ & $\begin{array}{c}26.80 \\
(26.78)\end{array}$ & - \\
\hline $2 f$ & $-\mathrm{N}\left(\mathrm{CH}_{3}\right)_{2}$ & 274.32 & $\mathrm{C}_{13} \mathrm{H}_{18} \mathrm{~N}_{6} \mathrm{O}$ & 161 & $\begin{array}{c}56.92 \\
(56.90)\end{array}$ & $\begin{array}{c}6.61 \\
(6.60)\end{array}$ & $\begin{array}{c}30.64 \\
(30.61)\end{array}$ & - \\
\hline $2 \mathrm{~g}$ & $-\mathrm{CH}_{3}$ & 245.28 & $\mathrm{C}_{12} \mathrm{H}_{15} \mathrm{~N}_{5} \mathrm{O}$ & 154 & $\begin{array}{c}58.76 \\
(58.77) \\
\end{array}$ & $\begin{array}{c}6.16 \\
(6.18)\end{array}$ & $\begin{array}{c}28.55 \\
(28.51) \\
\end{array}$ & - \\
\hline
\end{tabular}

Table 2: Antimicrobial activities of compounds 1a-g and 2a-g at $100 \mu \mathrm{g} / \mathrm{mL}$

\begin{tabular}{|c|c|c|c|c|c|c|c|c|c|c|}
\hline \multirow[b]{2}{*}{ Compound } & \multicolumn{5}{|c|}{ Antibacterial screnning } & \multicolumn{5}{|c|}{ Antifungal screnning } \\
\hline & S. a & E.c & E.f & P. $a$ & K.p & A.n & C.a & A.f & C.n & M.a \\
\hline $1 \mathrm{a}$ & 12 & - & - & 12 & - & 7 & - & - & - & 14 \\
\hline $1 \mathrm{~b}$ & 28 & 8 & 10 & 10 & 12 & 16 & 24 & 15 & 7 & 13 \\
\hline 1c & 16 & - & - & 16 & 19 & 10 & 6 & - & - & 8 \\
\hline 1d & 20 & - & 12 & 18 & 8 & - & 7 & - & 16 & 6 \\
\hline $1 e$ & 18 & 12 & - & 12 & 10 & 17 & 26 & - & 7 & 8 \\
\hline $1 f$ & 15 & - & 14 & 14 & - & - & 15 & - & 9 & - \\
\hline $1 \mathrm{~g}$ & - & 24 & 25 & 26 & 12 & 9 & 17 & - & 16 & - \\
\hline $2^{a}$ & 10 & 12 & - & - & 10 & - & 7 & - & 9 & - \\
\hline 2b & 5 & - & - & 10 & 12 & 15 & 10 & 8 & 10 & 8 \\
\hline 2c & - & - & 12 & 12 & 8 & - & - & - & - & 16 \\
\hline $2 d$ & 12 & 10 & 10 & 15 & - & 10 & 16 & 14 & - & 20 \\
\hline $2 e$ & - & 16 & - & 28 & - & - & 12 & 6 & 8 & - \\
\hline $2 \mathrm{f}$ & 22 & 24 & 25 & - & 10 & - & 16 & - & 23 & - \\
\hline $2 \mathrm{~g}$ & 12 & - & 10 & 26 & 19 & 18 & 28 & 11 & 20 & 10 \\
\hline Ciprofloxacin & 26 & 28 & 22 & 15 & 19 & 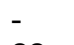 & - & - & - & - \\
\hline Clotrimazole & - & - & - & - & - & 22 & 26 & 16 & 30 & 18 \\
\hline
\end{tabular}

Clotrimazole was used as a standard; zone of inhibition was measured in $\mathrm{mm}$ 
Table 3: Minimum inhibitory concentrations (MIC, $\mu \mathrm{g} / \mathrm{mL}$ ) of compounds 1a-g and 2a-g

\begin{tabular}{|c|c|c|c|c|c|c|c|c|c|c|}
\hline \multirow{3}{*}{ Comp. No. } & \multicolumn{10}{|c|}{ Minimum Inhibitory Concentration (MIC, $\mu \mathrm{g} / \mathrm{mL})^{\mathrm{a}}$} \\
\hline & \multicolumn{5}{|c|}{ Antibacterial activity } & \multicolumn{5}{|c|}{ Antifungal activity } \\
\hline & S.a & E.c & E.f & P.a & K.p & A.n & C. $a$ & A.f & C. $n$ & M. a \\
\hline 1b & 0.25 & - & - & 32 & - & 32 & 4 & 64 & - & $>100$ \\
\hline 1c & 16 & - & - & 16 & 4 & $>100$ & $>100$ & - & - & $>100$ \\
\hline 1d & 2 & - & 64 & 1 & $>100$ & - & $>100$ & - & 64 & $>100$ \\
\hline $1 \mathrm{~g}$ & - & 1 & 0.5 & 0.25 & 64 & 32 & 1 & - & $>100$ & $>100$ \\
\hline $2 d$ & 64 & 64 & 64 & 16 & - & 64 & 0.25 & 64 & - & 1 \\
\hline $2 e$ & - & 4 & - & & 0.5 & - & 32 & $>100$ & $>100$ & - \\
\hline $2 f$ & 2 & 0.5 & 0.5 & - & 64 & - & 16 & - & 2 & - \\
\hline $2 g$ & 64 & - & 64 & 0.5 & 2 & 4 & 0.5 & $>100$ & 2 & 32 \\
\hline Ciproflaxacin & 0.5 & 0.5 & 0.5 & 4 & 2 & - & - & - & - & - \\
\hline Clotrimazole & - & - & - & - & - & 2 & 1 & 8 & 0.5 & 2 \\
\hline
\end{tabular}

Table 4: Larvicidal profile of compounds $1 \mathbf{a}-\mathbf{g}$ and $\mathbf{2 a - g}$ on against second instar larvae of Culex sp

\begin{tabular}{|c|c|c|c|c|c|}
\hline \multirow[t]{2}{*}{ Compound no. } & \multicolumn{4}{|c|}{$\begin{array}{c}\text { Mortality }(\%) \text { at room temp } \\
\text { concentration }(\mu \mathrm{g} / \mathrm{mL})\end{array}$} & \multirow{2}{*}{$\begin{array}{c}\mathrm{LD}_{50} \\
(\mu \mathrm{g} / \mathrm{mL})\end{array}$} \\
\hline & 10 & 20 & 30 & 40 & \\
\hline $1^{a}$ & $25 \pm 4.8$ & $51 \pm 3.1$ & $79 \pm 8.2$ & $100 \pm 0.0$ & 19.5 \\
\hline $1 b$ & $22 \pm 3.7$ & $75 \pm 4.8$ & $90 \pm 2.5$ & $100 \pm 0.0$ & 16.2 \\
\hline 1c & $29 \pm 4.1$ & $54 \pm 40$ & $100 \pm 0.0$ & - & 16.9 \\
\hline $1 d$ & $18 \pm 1.2$ & $51 \pm 1.2$ & $76 \pm 4.2$ & $100 \pm 0.0$ & 20.8 \\
\hline $1 e$ & $45 \pm 2.9$ & $86 \pm 2.5$ & $100 \pm 0.0$ & - & 10.1 \\
\hline $1 f$ & $27 \pm 4.8$ & $64 \pm 4.0$ & $82 \pm 4.2$ & $100 \pm 0.0$ & 17.2 \\
\hline $1 \mathrm{~g}$ & $52 \pm 4.1$ & $73 \pm 4.2$ & $100 \pm 0.0$ & - & 9.5 \\
\hline $2 a$ & $20 \pm 2.2$ & $59 \pm 7.3$ & $74 \pm 3.8$ & $100 \pm 0.0$ & 19.8 \\
\hline $2 b$ & $24 \pm 2.1$ & $40 \pm 3.8$ & $82 \pm 5.7$ & $100 \pm 0.0$ & 19.1 \\
\hline 2c & $20 \pm 3.2$ & $46 \pm 4.8$ & $60 \pm 3.9$ & $100 \pm 0.0$ & 22.4 \\
\hline $2 d$ & $10 \pm 3.2$ & $32 \pm 2.5$ & $66 \pm 3.8$ & $100 \pm 0.0$ & 24.3 \\
\hline $2 e$ & $28 \pm 3.1$ & $62 \pm 4.0$ & $100 \pm 0.0$ & - & 16.2 \\
\hline $2 f$ & $22 \pm 3.7$ & $63 \pm 3.5$ & $71 \pm 1.7$ & $100 \pm 0.0$ & 19.2 \\
\hline $2 g$ & $30 \pm 4.8$ & $58 \pm 3.8$ & $92 \pm 4.2$ & $100 \pm 0.0$ & 16.8 \\
\hline
\end{tabular}

Values are mean $\pm S D(n=3)$

Table 5: Nematicidial activities of compounds 1a-g and 2a-g

\begin{tabular}{|c|c|c|c|c|c|}
\hline \multirow[t]{2}{*}{ Compound no. } & \multicolumn{4}{|c|}{$\begin{array}{c}\text { Mortality }(\%) \text { at room temp } \\
\text { concentration }(\mu \mathrm{g} / \mathrm{mL})\end{array}$} & \multirow{2}{*}{$\begin{array}{c}L_{50} \\
(\mu \mathrm{g} / \mathrm{mL})\end{array}$} \\
\hline & 10 & 20 & 30 & 40 & \\
\hline $1 \mathrm{a}$ & $20 \pm 3.8$ & $49 \pm 3.7$ & $87 \pm 3.2$ & $100 \pm 0.0$ & 20.6 \\
\hline $1 b$ & $40 \pm 4.1$ & $51 \pm 2.3$ & $80 \pm 1.9$ & $100 \pm 0.0$ & 16.5 \\
\hline 1c & $39 \pm 4.1$ & $76 \pm 2.3$ & $100 \pm 0.0$ & - & 12.8 \\
\hline $1 d$ & $43 \pm 4.8$ & $71 \pm 5.7$ & $100 \pm 0.0$ & - & 12.5 \\
\hline $1 e$ & $42 \pm 4.0$ & $61 \pm 1.8$ & $100 \pm 0.0$ & - & 13.9 \\
\hline $1 f$ & $42 \pm 4.1$ & $53 \pm 4.1$ & $100 \pm 0.0$ & - & 14.8 \\
\hline $1 \mathrm{~g}$ & $54 \pm 4.8$ & $72 \pm 2.3$ & $100 \pm 0.0$ & - & 8.9 \\
\hline $2 a$ & $18 \pm 2.1$ & $36 \pm 4.4$ & $60 \pm 2.0$ & $100 \pm 0.0$ & 23.7 \\
\hline $2 b$ & $30 \pm 3.2$ & $56 \pm 3.2$ & $72 \pm 3.9$ & $100 \pm 0.0$ & 18.5 \\
\hline $2 c$ & $38 \pm 1.9$ & $40 \pm 3.8$ & $60 \pm 2.0$ & $100 \pm 0.0$ & 20.3 \\
\hline $2 d$ & $23 \pm 5.0$ & $52 \pm 4.1$ & $100 \pm 0.0$ & - & 17.8 \\
\hline $2 e$ & $42 \pm 4.1$ & $66 \pm 2.2$ & $100 \pm 0.0$ & - & 13.3 \\
\hline $2 f$ & $28 \pm 3.0$ & $47 \pm 2.8$ & $62 \pm 2.0$ & $100 \pm 0.0$ & 20.9 \\
\hline $2 g$ & $16 \pm 1.2$ & $45 \pm 4.1$ & $67 \pm 3.9$ & $100 \pm 0.0$ & 22.4 \\
\hline
\end{tabular}

Values are the means of three replicates $\pm S D$ 


\section{DISCUSSION}

We synthesised and characterised 14 new Mannich base imidazole derivatives (1a-g) and $(2 \mathbf{a}-\mathbf{g})$ as outlined in Scheme 1. The physical data of these compounds are given in Table 1.

The Mannich base condensation reaction proceeds via an attack by benzaldehyde at a secondary amine. During this reaction, one mole of water was eliminated and the resulting thiazolidine-4-one products were purified by column chromatography using an eluent of hexane: chloroform (1:4)

The chemical structure of each new compound was confirmed by IR, ${ }^{1} \mathrm{H}$ NMR, ${ }^{13} \mathrm{C}$ NMR, mass, and elemental analysis. The IR spectrum of compound 1a showed absorption bands at $3292.26,2916.17,1436.87$ and $460.03 \mathrm{~cm}^{-1}$ corresponding to $\mathrm{NH}_{2}, \mathrm{NH}, \mathrm{C}=\mathrm{S}$ and $\mathrm{CH}$ groups. The ${ }^{1} \mathrm{H}$ NMR spectrum of the compounds 1a showed broad signals at 9.53, 7.83 and 2.20 ppm corresponding to $\mathrm{NH}_{2}, \mathrm{CH}$ and $\mathrm{NH}$ protons respectively. The ${ }^{13} \mathrm{C}$ NMR spectra of 1 a contained important peaks at 182.7 and 75.3 ppm, corresponding to $\mathrm{C}=\mathrm{S}$ and $\mathrm{CH}$ carbon atoms, respectively. The mass spectrum of 1 a contained a molecular ion peak $\mathrm{m} / \mathrm{z} 247.28$, thereby confirming its molecular mass. Similar spectral data and corresponding molecular masses were obtained for compounds (1b-1g).

Similarly, the IR spectrum of compound $2 \mathbf{a}$ showed absorption bands at 3287.20, 2932.97, 1623.21 and $456.98 \mathrm{~cm}^{-1}$ corresponding to $\mathrm{NH}_{2}$, $\mathrm{NH}, \mathrm{C}=\mathrm{O}$ and $\mathrm{CH}$ groups respectively. The ${ }^{1} \mathrm{H}$ NMR spectrum of $\mathbf{2 a}$ showed broad signals at 9.50, 7.86 and $2.21 \mathrm{ppm}$, corresponding to $\mathrm{NH}_{2}$, $\mathrm{CH}$ and $\mathrm{NH}$ protons respectively. The ${ }^{13} \mathrm{C} N M R$ spectrum of compound $\mathbf{2 a}$ showed important peaks at 157.9 and $75.9 \mathrm{ppm}$, corresponded to
$\mathrm{C}=\mathrm{O}$ and $\mathrm{CH}$ carbon atoms respectively. The mass spectrum of $\mathbf{2 a}$ contained a molecular ion peak $\mathrm{m} / \mathrm{z} 231.28$, which is consistent with its molecular mass. Similar spectral data and corresponding molecular masses were obtained for compounds (2b-2g).

The synthesized imidazole derivatives were evaluated in terms of their antimicrobial, larvicidal and, nematicidal activities. Consistent with their expected structure - activity relationship, compounds $\mathbf{1 a - 1 g}$ and $\mathbf{2 a - 2 g}$ were biologically active. The presence of imidazole nucleus and the para substitution of the phenyl ring contribute to the observed activities.

The chemical structure in Figure 1 show that the 4-substituted phenyl ring acts as a lipophilic domain. The $\mathrm{C}=\mathrm{S}$ group in thiosemicarbazone and the $\mathrm{C}=\mathrm{O}$ group in semicarbazone form hydrogen bonds with the $\mathrm{NH}$ groups in thiosemicarbazone and semicarbazone act as hydrogen bonding domain. Therefore, the imidazole ring is an essential pharmacophore that determine biological activity.

Compound 1b, which contains a 4-substituted chlorine atom showed significant antibacterial activity against $S$. aureus (MIC, $0.25 \mu \mathrm{g} / \mathrm{mL}$ ) relative to that of the positive control ciproflaxiacin (MIC: $0.5 \mu \mathrm{g} / \mathrm{mL}$ ).

Compound $\mathbf{1 g}$, with a 4-substituted methyl group, exhibited a remarkable activity against $P$. aeruginosa (MIC, $0.25 \mu \mathrm{g} / \mathrm{mL}$ ) compared with that of ciprofloxacin (MIC, $4 \mu \mathrm{g} / \mathrm{mL}$ ).

Compound 2d containing a 4- $\mathrm{NO}_{2}$ group exhibited an excellent antifungal activity (MIC, $0.25 \mu \mathrm{g} / \mathrm{mL}$ ) against $C$. albicans relative to the activities of the synthesized compounds and the positive control, clotrimazole (MIC, $1 \mu \mathrm{g} / \mathrm{mL}$ ).

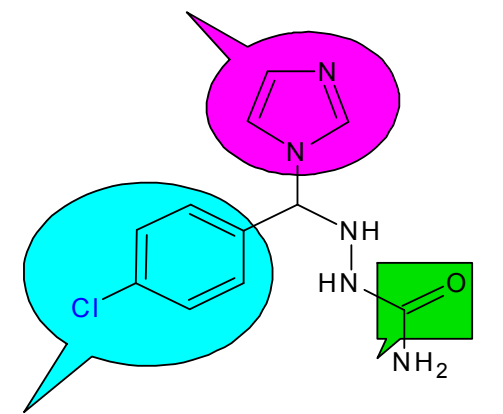

Compound 1b

Staphylococcus aureus MIC: $0.25 \mu \mathrm{g} / \mathrm{mL}$

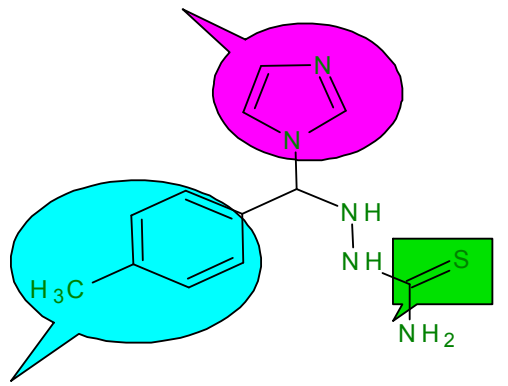

Compound $\mathbf{1 g}$

Pseudomonas aeruginosa :MIC: $0.25 \mu \mathrm{g} / \mathrm{mL}$ Nematicidal activity $L_{50}=8.9 \mu \mathrm{g} / \mathrm{mL}$ Larvicidal activity LD $_{50}=9.5 \mu \mathrm{g} / \mathrm{mL}$

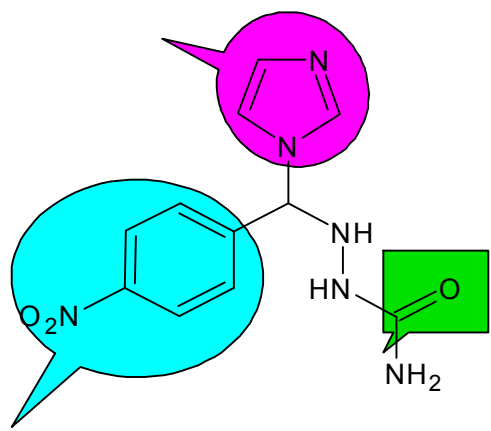

Compound 2d

Candida albicans, MIC:0.25 $\mu \mathrm{g} / \mathrm{mL}$

Figure 1: Structure activity relationships of synthesised imiazole derivatives 
Compound $1 \mathrm{~g}$, which contains a $4-\mathrm{CH}_{3}$-phenyl group with thiosemicarbazone and imidazole moieties, was a potent larvicide $\left(\mathrm{LD}_{50}: 9.5\right.$ $\mu \mathrm{g} / \mathrm{mL})$ and nematicide $\left(\mathrm{LD}_{50}, 8.9 \mu \mathrm{g} / \mathrm{mL}\right)$ while compound $\mathbf{2 g}\left(4-\mathrm{CH}_{3}\right.$-phenyl with semicarbazide and imidazole moieties) showed no significant nematicidal $\left(\mathrm{LD}_{50}, 22.9 \mu \mathrm{g} / \mathrm{mL}\right)$ or larvicidal $\left(\mathrm{LD}_{50}, 16.8 \mu \mathrm{g} / \mathrm{mL}\right)$ activities.

Compound 1e, which also contains a $4-\mathrm{CH}_{3} \mathrm{O}-$ phenyl with thiosemicarbazone and imidazole moieties, was also a potent larvicide $\left(\mathrm{LD}_{50}, 10.1\right.$ $\mu \mathrm{g} / \mathrm{mL})$ and nematicide $\left(\mathrm{LD}_{50}, 13.9 \mu \mathrm{g} / \mathrm{mL}\right)$, while compound $2 \mathrm{e}$ was less active against mosquito larvae $\left(\mathrm{LD}_{50}, 16.2 \mu \mathrm{g} / \mathrm{mL}\right)$ and highly active against nematodes $\left(\mathrm{LD}_{50}, 13.9 \mu \mathrm{g} / \mathrm{mL}\right)$. However, compound $1 \mathrm{~g}$ exhibited the highest activity in both cases.

\section{CONCLUSION}

A new series of imidazole derivatives has been synthesized. Some of them possess strong larvicidal, nematicidal, antibacterial and antifungal activities, and thus are capable of serving as potential lead molecules for the development of clinically useful antimicrobial, larvicidal and nematicidal agents.

\section{REFERENCES}

1. Grimmett MR. Advances in Heterocyclic Chemistry. New York, 1970; 27: pp 241-326.

2. Jouneau S, Bazureau JP. Solvent free aza-annulation using 4-dimethylamino 2-aza-1,3-dienes as $\gamma$ dielectrophiles for a new synthesis of imidazole-4carboxylates. Tetrahedron Lett 1999; 40: 8097-8098.

3. Lambardino JG, Wiseman EH. Preparation and antiinflammatory activity of some nonacidic trisubstituted imidazoles. J Med Chem 1974; 17: 1182-1188.

4. Maier T, Schmierer R, Bauer K, Bieringer $H$, Buerstel $H$, Sachse B. 1-substituted imidazole-5-carboxylic acid derivatives, their preparation and their use as biocides. U.S. Patent, 820335, 1989; US, Chem Abstr 1989, 19494

5. De Luca L. Naturally occurring and synthetic imidazoles: their chemistry and their biological activities. Curr Med Chem 2006; 13: 1-23.
6. Boiani M, González M. Imidazole and benzimidazole derivatives as chemotherapeutic agents. Mini-Rev Med Chem 2005; 5: 409-424.

7. Verma M, Chaturved AK, Chowdhari A, Parmar SS. Monoamine oxidase inhibitory and anticonvulsant properties of 1,2,4-trisubstituted 5-imidazolones. J Pharm Sci 1974; 63: 1740-1744.

8. Harfenist M, Saroka FE, Meckenzie GM. 2-(Alkoxyaryl)-2imidazoline monoamine oxidase inhibitors with antidepressant activity. J Med Chem 1978; 21: 405409.

9. Mannich C, Krosche W. Ueber ein Kondensationsprodukt aus Formaldehyd, Ammoniakund Antipyrin. Archiv der Pharmazie 1912; 250: 647-667.

10. Tramontini $M$, Angliolini $L$. Further advances in the chemistry of mannich bases Tetrahedron 1990; 46: 1791-1837

11. Thompson BB. The Mannich reaction. Mechanistic and technological considerations. J Pharm Sci 1968; 57: 715-733.

12. Siatra-Papastaikoudi T, Tsotinis A, Chinou I, Roussakis C. Synthesis and anticancer activity of new phenylring substituted 4-morpholino-1-phenylthio-2butanones Mannich bases. Farmaco 1994; 49: 221 223.

13. Koechel DA, Rankin GO. Diuretic activity of Mannich base derivatives of ethacrynic acid and certain ethacrynic acid analogues. J Med Chem 1978; 21: 764-769.

14. Lee CM, Plattner JJ, Ours CW, Horrom BW, Smital JR, Pernet AG, Bunnell PR, El-Masry SE, Dodge PW. Aminomethyl aryl oxy acetic acid esters. A new class of high-ceiling diuretics. 1. Effects of nitrogen and aromatic nuclear substitution. J Med Chem 1984; 27 : 1579-1587.

15. Bauer AW, Kirby WM, Sherris JC, Turck JC. M. Antibiotic susceptibility testing by a standardized single disk method. Am Clin Pathol 1966; 39(5): 493-496.

16. Petersdorf RG, Sherris JC. Methods and significance of in vitro testing of bacterial sensitivity to drugs. Am J Med 1965; 39(5): 766-779.

17. Gillespie S H. "Medical Microbiology-Illustrated," Butterworth Heinemann, London, 1994, 234-237pp. In Varma RS. Editor, Antifungal Agents: Past, Present and Future prospects, National Academy of Chemistry \& Biology, Lucknow, India, 1998.

18. Manilal A, Sujith S, Kiran GS, Selvin J. Shakir C, Gandhimathi R. Biopotential of seaweeds sollected from Southwest coast of India. J Marine Sci Techno 2009; 17: 67-73. 